\title{
The influence of Urbanisation on Phytodiversity and some edaphic properties in urban and peri-urban riverine fresh water wetlands of Bamenda Municipality
}

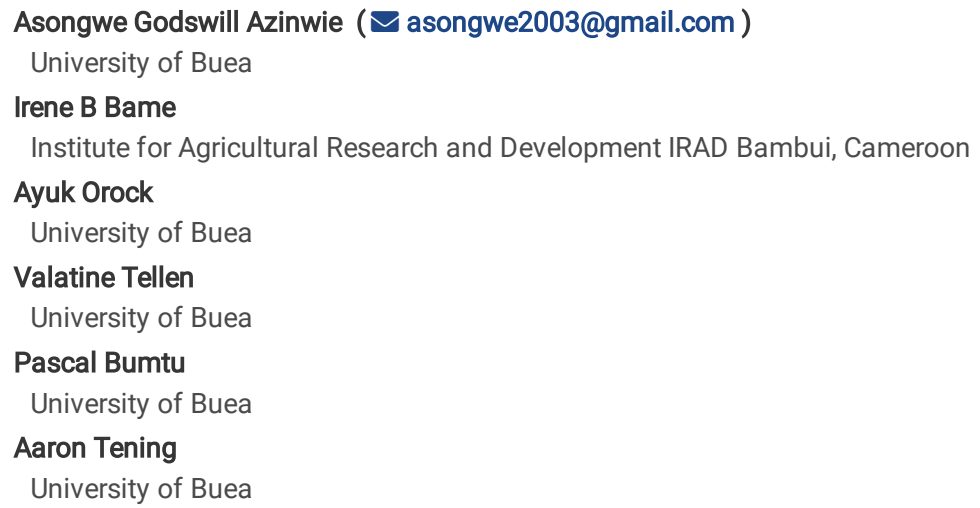

Research Article

Keywords: Species diversity, soil properties, urbanization, Bamenda municipality.

Posted Date: March 7th, 2022

DOl: https://doi.org/10.21203/rs.3.rs-1405544/v1

License: (c) (i) This work is licensed under a Creative Commons Attribution 4.0 International License. Read Full License 


\section{Abstract}

Aims

In urban areas, human activities result to the discharge of a variety of chemical substances to the environment, playing a substantial role in soil quality, plant species diversity and human security. In order to suggest appropriate management strategies that ensures soil quality, their sustainable utilization and human security in the midst of urbanization, this study assesses the relationship between macrophyte diversity and some soil characteristics of wetlands in Bamenda Municipality, Cameroon at different stages of urbanization. It examines (i) macrophyte diversity and soil characteristics of wetlands adjacent urban and periurban areas.

\section{Methods}

Plant communities were sampled for species composition and relative abundance, using the Braun-banquet method. Species richness was evaluated using the Simpson's diversity index. Twenty-one soil samples (0-25 cm depth) were also randomly collected within the wetlands and analyzed for their physicochemical characteristics using standard methods. The hierarchical cluster analysis (HCA) was used to group the area under managing units.

\section{Results}

From the results, 50 macrophytes species distributed in 28 families were documented in the area. The species observed were mostly emergent herbaceous plants (grasses) with only few shrubs and trees. The Simpson indices of diversity were 0.94 and 0.96 for the urban and control sites, respectively. The soils were slightly acidic. Sand, $\mathrm{pH}-\mathrm{H}_{2} \mathrm{O}, \mathrm{pH}-\mathrm{KCl}$, Na were consistently least variable across the three sites. Two significant clusters representing a combination of urban and peri urban/rural were formed from the hierarchical dendrograms for classification of the chemical variables for the surface soils wetlands with associations to plants species. The Mann-Whitney $U$ test, revealed a significant lower $(P<0.05)$ concentration of the chemical constituents of the control site and the urban sites indicating contamination.

\section{Conclusion}

Intensification and extension of urbanisation is reducing the diversity of the wetlands of the Bamenda municipality warranting monitoring

\section{Introduction}

Human activities either direct and/or indirect ranging from global warming, landscape alteration, agriculture and urbanisation represent an existential problem between nature and society in relation to phytodiversity and soil quality (Solbrig, 2000; Pena-Claros et al., 2012; Arruda et al., 2015; Germ et al., 2021). Phytodiversity is one of the indicators often used to measure wellbeing of ecological systems (Borgwardt et al., 2019). In tropical regions, the structure and diversity of vegetation are determined by several biotic and/or abiotic factors, which act in different spatial and temporal scales (Dale, 1999).

Topography and soil type are among the major abiotic factors that play a major role in the heterogeneity of habitats and species, thus contributing to physiognomic differentiation of the vegetation (Baldeck et al., 2013; Guerra et al., 2013).

In urban areas, human activities results to the discharge of a variety of chemical substances to the environment that play a substantial role in altering the quality of the soil. This ultimately results in changes on the structure of plant community, their species diversity and human security.

According to Yerima and Van Ranst (2005), plants and soils are intricately related to an extent that changes in any of them would alter the other significantly. This could be partly associated to the fact that the fertility of soils is strongly influenced by the types of plants that grow. The types of plants influence the development of soil profiles (Yerima and Van Ranst, 2005).

Wetlands in urban and peri-urban areas can provide a range of important ecosystem services and benefits to people. However, in many countries, just as in Cameroon, as a result of spreading urbanization, wetlands are increasingly becoming degraded. The deposition of wastes in these zones also defies the Cameroon law on the Environment, Section III article 31(1) that forbids the deposition of waste into natural drains (MINEP, 1996). Todd (1980) and Malida (1981) observed that industrial and domestic wastes deposited in water add large amounts of organic and inorganic substances ranging from trace metals, to acids and alkalis that are potential pollutants in aquatic systems. The deposition of wastes into these systems therefore portends danger-necessitating studies. Macrophytes are important components of wetlands where they grow vigorously in enriched areas with a species variety that is strongly related to the chemical constituents of the environment. Indeed, macrophytes have high remediation potentials for macro and micronutrients because of their general fast growth and high biomass production in contaminated areas. In this regard, emergent and floating species especially those commonly used in the treatment of wastewater (Greenway, 2003) are good indicators of environmental degradation in wetlands.

This study assesses the relationship between macrophyte diversity and some soil characteristics (physical and chemical) of wetlands in Bamenda Municipality, Cameroon at different stages of urbanization.

It is hypothesized that (i) macrophyte diversity and soil soil characteristics do not differ among the wetlands originating from different ages of urbanization, (ii) soil characteristics do not influence the vegetation structure and plant diversity. These findings would help to suggest appropriate management strategies that will ensure soil quality, their sustainable utilization and human security in the midst of urbanization.

\section{Materials And Methods}




\section{Description of the study area}

Geographically, this study covers urban and peri-urban wetlands in the Bamenda City Council of the North West Region of Cameroon that have evolved concomitantly with different stages of urbanization (Figure 1). The area is part of the Bamenda escarpment located between latitudes $5^{\circ} 55^{\prime \prime} \mathrm{N}$ and $6^{\circ} 30^{\prime \prime} \mathrm{N}$ and longitudes $10^{\circ} 25^{\prime \prime} \mathrm{E}$ and $10^{\circ} 67^{\prime \prime} \mathrm{E}$. The town shows an altitudinal range of $1200-1700 \mathrm{~m}$, and is divided into two parts by escarpments; a low lying gently undulating part with altitudes ranging from 1200 to $1400 \mathrm{~m}$, with many flat areas that are usually inundated for most parts of the year, and an elevated part at 1400 to $1700 \mathrm{~m}$ altitude that forms the crest from which creeks, and streams, supplying the low lying parts take their rise.

This area experiences two seasons; a long rainy season, (mid-March to mid-October) and a short dry season (mid-October to mid-March). The thermic and hyperthemic temperature regimes dominate in the area. Mean annual temperatures stand at $19.9^{\circ} \mathrm{C}$. January and February are the hottest months with mean monthly temperatures of $29.1^{\circ} \mathrm{C}$ and $29.7^{\circ} \mathrm{C}$, respectively. This area is dominated by the Ustic and Udic moisture regimes with the Udic extending to the south (Yerima and Van Ranst, 2005). Annual rainfall ranges from 1300 - 3000 mm (Ndenecho, 2005). The area has a rich hydrographical network with intense human activities and a dense population along different water courses in the watershed. The area is bounded on the West, North and East by the Cameroon Volcanic Line (made up of basalts, trachytes, rhyolites and numerous salt springs). The geologic history of this area originates from the Precambrian era where there was vast formation of geosynclinal complexes, which became filled by clay-calcareous, and sandstone sediments (Yerima and Van Ranst, 2005). These materials, crossed by intrusions of crystalline rocks, were folded in a generally NE-SW direction and underwent variable metamorphism (Yerima and Van Ranst, 2005). The Rocks in the area are thus of igneous (granitic and volcanic) and metamorphic (migmatites) origin (Kips et al., 1987), which give rise to ferralitic soils (Grass field Participatory-Decentralised and Rural Development Project: GP- DERUDEP, 2006).

Agriculture is the principal human activity in and around this (GP-DERUDEP, 2006). The area equally harbours the commercial centre that has factories ranging from soap production, and mechanic workshops to metallurgy, which may be potential sources of pollutants that can influence wetland Geochemistry. Raffia farinifera bush, largely limited to the wetlands (Valleys and depressions), is an important vegetation type in this area. R. farinifera provides raffia wine, a vital economic resource to the inhabitants who are fighting against the cultivation of these wetlands by vegetable farmers.

\section{Methods of the Study}

\section{Macrophyte diversity study}

Plant diversity of the wetlands was evaluated using quadrats in the dry season when wetlands are accessible. For each of the wetlands, transects were established on which representative plots were mapped out on each of them for the determination of plant species cover abundance and diversity. Each of these plots layed measured $10 \times 10 \mathrm{~m}\left(100 \mathrm{~m}^{2}\right)$ and was established in an uncultivated area. The plant communities were sampled for species composition and relative abundance. The Braun-banquet method was used (Braun-Blauquet, 1965) for the assessment of species cover abundance. Relative abundance and abundance ratings were determined using the Braun-Blanquet rating scheme (Table 1). Diversity studies involved the identification of a specific area called "relevé" by progressively increasing test quadrat size and sampling for specific diversity until the smallest area with adequate species representation was reached. The relevé size determined here was $1 \mathrm{~m}^{2}$. For each site, 10 relevés were sampled and all plants species were enumerated. Most plant species in each of them were identified on the spot. For unidentified plants, appropriately collected were placed on to a plant press dried and identified in the herbarium of the Limbe Botanic Garden, using standard botanical techniques.

\section{Simpson's diversity index}

Species richness was evaluated using Simpson's diversity index. This index of diversity is more representative in pollution studies than the Shanon and Wiener index of diversity. Simpson's index (D) is a measure of diversity, which takes into account both species richness, and Table 1: Braun-Blanquet rating scheme for vegetation cover abundance and evenness of abundance among the species present. In essence it measures the probability that two individuals randomly selected from an area will belong to the same species. The formula for calculating $D$ is presented as:

Table 1. Braun-Blanquet rating scheme for vegetation cover abundance.

\begin{tabular}{|llll|}
\hline Class & \% range of cover & Mean & Rating \\
\hline 5 & $75-100$ & 87.5 & Very numerous \\
\hline 4 & $50-75$ & 62.5 & Numerous \\
\hline 3 & $25-50$ & 37.5 & Not numerous \\
\hline 2 & $5-25$ & 15.0 & Occasional \\
\hline 1 & $1-5$ & 2.5 & Sparse \\
\hline $\mathrm{R}$ & $<1$ & - & - \\
\hline
\end{tabular}

Source: Braun-Blauquet, 1965

$$
\mathrm{D}=\frac{\sum \mathrm{n}_{i}\left(\mathrm{n}_{i}-1\right)}{\mathrm{N}(\mathrm{N}-1)}
$$

where $\mathrm{n}_{i}=$ the total number of each individual species 
$\mathrm{N}=$ the total number of individuals of all species

The value of $\mathbf{D}$ ranges from 0 to 1 . With this index, 0 represents infinite diversity and, 1 , no diversity. That is, the bigger the value the lower the diversity.

Alternatively, Simpson's Diversity Index, = 1 - D,

1-D rather than $\mathrm{D}$ used as a measure of diversity; As it's more logical and less likely to cause confusion. The scale then gives a score from 0 to 1 with higher scores showing higher diversity (instead of being associated with low scores).

The Simpson index is a dominance index because it gives more weight to common or dominant species. In this case, a few rare species with only a few representatives will not affect the diversity.

\section{Soil sampling and analysis}

Twenty-one soil samples $(0-25 \mathrm{~cm})$ were randomly collected within the wetlands (Figure 1$)$ and taken to the laboratory in black plastic bags. The soil samples were air-dried and screened through a 2-mm sieve. They were analyzed for routine parameters in the Environmental and Analytical Chemistry Laboratory of the University of Dschang, Cameroon. Particle size distribution, cation exchange capacity (CEC), exchangeable bases, electrical conductivity (EC) and pH were determined by standard procedures (Pauwels et al., 1992). Soil pH was measured both in water and $\mathrm{KCl}$ (1:2.5 soil/water mixture) using a glass electrode $\mathrm{pH}$ meter. Part of the soil was ball-milled for organic carbon (OC) (Walky and Black method) and Kjeldahl-N as largely described by Pauwels et al., (1992). Available $\mathrm{P}$ was determined by Bray I method. Exchangeable cations were extracted using $1 \mathrm{~N}$ ammonium acetate at pH 7. Potassium (K) and sodium $(\mathrm{Na})$ in the extract were determined using flame photometer and magnesium $(\mathrm{Mg})$ and calcium (Ca) were determined by complexiometric titration. Exchangeable acidity was extracted with $1 \mathrm{M} \mathrm{KCl}$ followed by quantification of Al and $\mathrm{H}$ by titration (Pauwels et al., 1992). Effective cation exchange capacity (ECEC) was determined as sum of bases and exchanged acidity.

Apparent CEC (CEC at pH7) was determined directly as outlined by Pauwels et al., (1992). Based on critical values of nutrients established for vegetables, nutrients were declared sufficient or deficient.

\section{Statistical analysis}

The data were subjected to statistical analysis using Microsoft Excel 2007 and SPSS statistical package 20.0. Soil properties were assessed for their variability using coefficient of variation (CV) and compared with variability classes (Tables 2).

Where: $\mathrm{Sd}=$ standard deviation; $=X$ arithmetic mean of soil properties.

Table 1. Grouping coefficient of variation into variability classes

\begin{tabular}{|ll|}
\hline . CV (\%) & Variability grouping (class) \\
\hline$<15$ & Slightly variable \\
\hline $15-35$ & Moderately variable \\
$>35$ & Highly variable \\
\hline
\end{tabular}

The hierarchical cluster analysis ( $\mathrm{HCA}$ ) was use to group the area under managing units. The hierarchical agglomerative cluster analysis main goal is to spontaneously classify the data into groups of similarity (clusters) searching objects in the n-dimensional space located in closest neighborhood and to separate a stable cluster from other clusters. Usually, the sampling sites are considered as objects for classification, each one determined by a set of variables (chemical concentrations of the soils in this case).

\section{Results}

\section{Macrophyte Biodiversity Study}

Semi aquatic and marshland plant species in the wetlands were mainly herbaceous and shrubby. Most of the plants showed discoloration in patches throughout the wetland (Figure 2). Human activities involving cutting, of raffias and woody species resulted to an open vegetation with shrubs scattered all over the wetlands.

Table 2 presents a checklist of plant diversity in agricultural wetlands in the Bamenda Municipality (Mile 4 - Foncha fish pond segment (MF): Ayaba Ngomegham segment (AN) and a control site at Mbelewa Nkwen (MB). Where: $-=$ absent $+=$ observed, $1=$ covering less than $5 \%$ of surface; $2=$ present covering at least $5-25 \%$ of surface; $3=$ present covering more than $25 \%$ of area).

Table 2: Checklist of macrophytes diversity in the wetland in Bamenda at Mile 4 - Foncha fish pond segment (MF): Ayaba - Ngomegham segment (AN) and a control site at Mbelewa Nkwen (MB) 


\begin{tabular}{|c|c|c|c|c|c|c|}
\hline \multirow{2}{*}{\multicolumn{2}{|c|}{ Family Species Name }} & \multirow[b]{2}{*}{ Class } & \multirow[b]{2}{*}{ Common name } & \multicolumn{3}{|c|}{ Abundance } \\
\hline & & & & $M F$ & AN & MB \\
\hline \multirow[t]{5}{*}{ Acanthaceae } & Brilantasia nitens Lindau. & & & 1 & + & 1 \\
\hline & Decliptera laxata C. B. Clarke & & & 1 & 1 & 1 \\
\hline & Justicia obliquifolia Standl. & Dicot & Blood medicine & + & + & + \\
\hline & Justicia flava (Forssk.) Vahl & Dicot & Blood medecine & - & - & 1 \\
\hline & Nesonia canenscens Var. Smithii & Dicot & - & - & + & + \\
\hline \multirow[t]{3}{*}{ Amaranthaceae } & Alternanthera sessalis $L$. & Dicot & Sessile joy weed & + & + & 1 \\
\hline & Amaranthus hybridus $L$ & Dicot & - & 1 & 1 & 1 \\
\hline & Amaranthus spinosus $L$ & Dicot & Spiny amaranth & 1 & 1 & + \\
\hline Apiaceae & Centella asiatica (L.) Urb. & Dicot & Pennywort & + & + & + \\
\hline Apocynaceae & Catharathus roseus & Dicot & Periiwinkle & - & - & 1 \\
\hline Araceae & Colocasia esculentus $L$ & Dicot & Cocoyam & + & + & + \\
\hline Arecaceae & Raffia farinifera Gaertn. (L) & Monocot & Raffia palm & 1 & + & 2 \\
\hline \multirow[t]{7}{*}{ Asteraceae } & Ageratum cornyzoides. $L$ & Dicot & King grass & 1 & 1 & 1 \\
\hline & Tithonia diversifolia Hemsl. & Dicot & Sun flower & 1 & 2 & - \\
\hline & Vernonia amygdalina Del & Dicot & Bitter leaves & 1 & 1 & + \\
\hline & Vernonia hymenolepis A. Rich & Dicot & Sweet bitter leaves & 1 & 1 & - \\
\hline & Vernonia gigantean (Walter) Trel. & Dicot & Wild large leaf & - & - & + \\
\hline & Spilanthes filicaulis (Schum. \& Thonn.) C. D. Adams & Dicot & Eye for fowl" & 1 & - & + \\
\hline & Synedrella nodiflora Cabi. & Dicot & Node weed & - & - & + \\
\hline Athyraceae & Diplazium zammati (Khhn) C. Chr. & Dicot & Single branch fern & - & - & + \\
\hline Casiapinaceae & Cassia alata Ls & Dicot & Leave like that of quava & - & + & - \\
\hline \multirow[t]{2}{*}{ Commelinaceae } & Commelina beghalensis $L$ & Dicot & - & 3 & 2 & 1 \\
\hline & Commelina diffusa (Burm) F & Dicot & Tropical sidewort & 1 & 1 & 1 \\
\hline \multirow[t]{2}{*}{ Convolvulaceae } & Ipomoes aquatic Forsk & Dicot & - & 1 & 1 & 1 \\
\hline & Ipomoea batatas $L$ & Dicot & Sweet potatoes & + & + & + \\
\hline Curcubitaceae & Zshneria scarbra (L.f.) Sond. & Dicot & Curcumber & - & + & - \\
\hline \multirow[t]{4}{*}{ Cyperaceae } & Cyperus haspen & Monocot & - & - & - & 1 \\
\hline & Cyperus distans & Monocot & - & 2 & 1 & 1 \\
\hline & Mariscus alternifolius (Sensu Hepper) & Monocot & - & - & - & 1 \\
\hline & Rhynchospora corymbosa (L.) Britton. & Monocot & - & - & - & 1 \\
\hline Dryopteridaceae & Dryopteris felix-mas(L) Schoot & Dicot & Fern plant & + & + & 1 \\
\hline \multirow[t]{2}{*}{ Fabaceae } & Desmodium salicifolium (Poir.) DC & Dicot & - & - & 1 & + \\
\hline & Centrosoma pubescens (D.C.) Benth & Dicot & - & - & + & 1 \\
\hline Malvaceae & Urena lobata $L$. & Dicot & - & 1 & - & 1 \\
\hline Maranthaceae & Marantchloa purpurea (Ridl.) Milne-Redh & Dicot & - & + & + & + \\
\hline Mimosaceae & Mimosa pudica & Dicot & - & - & + & - \\
\hline Nymphaeaceae & Nymphea alba $(H)$ & Dicot & Water lily & + & - & - \\
\hline Onagraceae & Ludwigia abysisnica A. Rich & Dicot & Water primerose & 1 & 1 & 2 \\
\hline Piperaceae & Piper umberlatum (L) & Dicot & - & - & - & + \\
\hline \multirow[t]{3}{*}{ Poaceae } & Anzonopus compressus (Sw.) P. Beauv. & Monocot & Fat leaf carpet grass & + & + & - \\
\hline & Coix lachrymal-jobi (L) & Monocot & - & 1 & - & 2 \\
\hline & Cyndon dactylon (Linn) & Monocot & Bermuda grass & 2 & 1 & + \\
\hline
\end{tabular}

Page 5/18 


\begin{tabular}{|c|c|c|c|c|c|c|}
\hline \multirow[t]{2}{*}{ Family } & \multirow[t]{2}{*}{ Name } & \multirow[b]{2}{*}{ Class } & \multirow[b]{2}{*}{ Common name } & \multicolumn{3}{|c|}{ Abundance } \\
\hline & & & & $M F$ & AN & $\mathrm{MB}$ \\
\hline & Digitaria sanguinalis $(L)$ & Monocot & Crabgrass & - & 1 & 1 \\
\hline & Echinochloa crus-pavonis(Kunth) Schult & Monocot & - & 1 & 1 & - \\
\hline & Echinochloa paramidelis (Lam.) Hitchc. & Monocot & - & 2 & 1 & 2 \\
\hline & Eragrostis spp. Michx. & Monocot & - & 1 & 1 & - \\
\hline & Imperata cylindica & Monocot & Spear grass & 1 & 1 & - \\
\hline & Leersia hexandra Sw. & Monocot & - & 2 & 2 & 2 \\
\hline & Panicum maximum Jacq. Var & Monocot & Guinea grass & 1 & 1 & - \\
\hline & Paspalum conjugatum P. J. Bergius & Monocot & Sour grass & 1 & - & 1 \\
\hline & Pennisetum purpereum (Schumach) & Monocot & Elephant grass & 1 & 2 & + \\
\hline & Saccharum officinarium (L) & Monocot & Sugar cane & - & 1 & - \\
\hline & Saciolepis Africana & Monocot & - & - & - & 1 \\
\hline Polygonaceae & Rumex nepalensis $L$. & Dicot & - & - & - & + \\
\hline Pteridiacea & Pteridium equilinum & Dicot & Braken fern & + & + & + \\
\hline Polygonaceae & Polygonum limbatum Meisn. & Dicot & - & 2 & 1 & 1 \\
\hline Portuleraceae & Portulaca oleraceae $L$ & Dicot & - & + & + & - \\
\hline Rubiaceae & Spermacoce latifoliaAubl. & Dicot & - & 1 & 1 & 1 \\
\hline Sellaginellaceae & Sellaginella Kraussiana (Kunze)A.Braun & Dicot & - & + & - & 1 \\
\hline Smilalacaceae & Smilax Krausiana Meisn & & - & - & - & 1 \\
\hline Solanaceae & Physalis angulate L. var. & & - & - & - & + \\
\hline Tiliaceae & Triumfetta cordifolia A. Rich. & & - & 1 & 1 & 1 \\
\hline Zingiberaceae & Aframomum officinalis Schum & Monocot & Ginger & + & - & 1 \\
\hline Total 30 & 50 & & & 34 & 34 & 39 \\
\hline
\end{tabular}

Macrophytes constituted an important part of the wetland. From the quadrat studies, 50 plant (macrophytes) species distributed in 28 families were identified in the wetlands (Figure 3).The species observed were mainly emergent herbaceous plants (grasses) with only few shrubs and trees. The Poaceae (26\%) occurred as the most represented family, followed by Asteraceae (12\%), Cyperaceae (8\%), Acanthaceae (6\%), Amaranthaceae (6\%) with other species less represented (Figure 10). The marsh land from visual observation was dominated by Leersia hexandra growing in open water and covering large areas.

The control site at Mbelewa was represented by 39 species. Here, the dominant species ranked in abundance were as follows Raphia farinifera > Ludwigia hexandra > Coix spp.> Leersia hexandra > Ehchinochloa paramidelis.

The Mile 4 to Below Foncha segment (Peri urban) was represented by 40 species. Here, the dominant species were ranked in abundance as Commelina bengalensis > Leersia hexandra > Cyperus distance > Ehchinochloa pyramidalis. At the Ayaba to Ngomegham segment Urban segment), Pennisetum purpureum $>$ Echinochloa pyramidalis $>$ Tithonia diversifolia $>$ Leersia hexandra were the abundant species. Pennisetum purpureum, Echinochloa pyramidalis and Tithonia diversifolia lined the main river course.

From the study, the Simpson indices of diversity were 0.94 each at Mulang (Table 3), and at Mile Four (Table 4), both the urban and peri-urban sites, lower than the 0.96 value recorded at Mbelewa, the control site (Table 5).

Table 3: Simpson index of diversity from vegetation sample at Mulang of the Bamenda wetland gardens 


\begin{tabular}{|c|c|c|c|c|c|c|}
\hline Family & Species & Class & Common name & Count (n) & $\mathrm{n}-1$ & $n(n-1)$ \\
\hline \multirow[t]{4}{*}{ Acanthaceae } & Brilantasia nitens Lindau & Dicot & - & 3 & 2 & 6 \\
\hline & Decliptera laxata C. B. Clarke & Dicot & - & 12 & 11 & 132 \\
\hline & Justicia obliquifolia Standl. & Dicot & Blood medicine & 2 & 1 & 2 \\
\hline & Nesonia canenscens Var. Smithii & Dicot & - & 5 & 4 & 20 \\
\hline \multirow[t]{3}{*}{ Amaranthaceae } & Alternanthera sessalis $L$. & Dicot & Sessile joy weed & 4 & 3 & 12 \\
\hline & Amaranthus hybridus $L$ & Dicot & - & 15 & 14 & 210 \\
\hline & Amaranthuss spinosus $L$ & Dicot & Spiny amaranth & 12 & 11 & 132 \\
\hline Apiaceae & Centella asiatica (L.) Urb. & Dicot & Penny wort & 5 & 4 & 20 \\
\hline Araceae & Colocasia esculentus $L$ & Dicot & Cocoyam & 3 & 2 & 6 \\
\hline Arecaceae & Raffia fanivera Gaertnb(L) & Monocot & Raffia palm & 3 & 2 & 6 \\
\hline \multirow[t]{4}{*}{ Asteraceae } & Ageratum cornyzoides. $L$ & Dicot & King grass & 12 & 11 & 132 \\
\hline & Tithonia diversifolia Hemsl. & Dicot & Sun flower & 35 & 34 & 1190 \\
\hline & Vernonia amygdalina Del & Dicot & Bitter leaf & 9 & 8 & 72 \\
\hline & Vernonia hymenolepis A. Rich & Dicot & Sweet bitter leaf & 4 & 3 & 12 \\
\hline Casiapinaceae & Cassia alata $L$. & Dicot & Leaves like that of guava & 2 & 1 & 2 \\
\hline \multirow[t]{2}{*}{ Commelinaceae } & Commelina beghalensis $L$ & Dicot & - & 43 & 42 & 1806 \\
\hline & Commelina diffusa (Burn) $F$ & Dicot & Tropical sidewort & 10 & 9 & 90 \\
\hline \multirow[t]{2}{*}{ Convolvulaceae } & Ipomoea aquatic Forsk & Dicot & - & 9 & 8 & 72 \\
\hline & Ipomea batatas $L$ & Dicot & Sweet potatoes & 5 & 4 & 20 \\
\hline Curcubitaceae & Zsheria scarbra (L.F.) Sond. & Dicot & Curcumber & 4 & 3 & 12 \\
\hline Cyperaceae & Cyperus distans & Monocot & - & 15 & 14 & 210 \\
\hline \multirow[t]{3}{*}{ Dryopteridaceae } & Dryopteris felix-mas(L) Schoot & Dicot & Fern plant & 2 & 1 & 2 \\
\hline & Centrosoma pubescens (D.C.) Benth & Dicot & - & 3 & 2 & 6 \\
\hline & Desmodium salicifolium (Poir.) DC & Dicot & - & 11 & 10 & 110 \\
\hline Maranthaceae & Marantchloa purpurea (Ridl.) Milne-Redh & Dicot & - & 1 & 0 & 0 \\
\hline Mimosaceae & Mimosa pudica & Dicot & - & 2 & 1 & 2 \\
\hline Onagraceae & Ludwigia abysisnica A. Rich & Dicot & Water primerose & 12 & 11 & 132 \\
\hline \multirow[t]{13}{*}{ Poaceae } & Anzonopus compressus (Sw.) P. Beauv. & Monocot & Flat leaf carpet grass & 7 & 6 & 42 \\
\hline & Cyndon dactylon (Linn) & Monocot & Bermuda grass & 16 & 15 & 240 \\
\hline & Digitaria sanguinalis $(L)$ & Monocot & Crab grass & 14 & 13 & 182 \\
\hline & Echinochloa crus-pavonis (Kunth) Schult & Monocot & - & 17 & 16 & 272 \\
\hline & Echinochloa paramidelis (Lam.) Hitchc & Monocot & - & 13 & 12 & 156 \\
\hline & Eragrostis spp. Michx. & Monocot & - & 10 & 9 & 90 \\
\hline & Imperata cylindica & Monocot & Spear grass & 15 & 14 & 210 \\
\hline & Leersia hexandra Sw. & Monocot & - & 67 & 66 & 4422 \\
\hline & Panicum maximum Jacq. Var & Monocot & Guinea grass & 10 & 9 & 90 \\
\hline & Pennisetum purpereum (Schumach) & Monocot & Elephant grass & 54 & 53 & 2862 \\
\hline & Saccharum officinarium (L) & Monocot & sugar cane & 10 & 9 & 90 \\
\hline & Pteridium equilinum & Dicot & Braken fern & 4 & 3 & 12 \\
\hline & Polygonium limbatum Meisn & Dicot & - & 12 & 11 & 132 \\
\hline Portuleraceae & Portulaca oleraceae $L$ & Dicot & - & 3 & 2 & 6 \\
\hline Rubiaceae & Spermacoce latifolia Aubl. & Dicot & - & 10 & 9 & 90 \\
\hline Total & 42 & & & 505 & & 13312 \\
\hline
\end{tabular}


$D=0.052302$

Simpson index $=0.94$

Table 4: Simpson index of diversity from vegetation samples at Mile Four. 


\begin{tabular}{|c|c|c|c|c|c|c|}
\hline Family & Species & Class & Common name & count (n) & $n-1$ & $n(n-1)$ \\
\hline \multirow[t]{3}{*}{ Acanthaceae } & Brilantasia nitens Lindau & Dicot & & 20 & 19 & 380 \\
\hline & Decliptera laxata C. B. Clarke & Dicot & - & 22 & 21 & 462 \\
\hline & Justicia obliquifolia Standl. & Dicot & Blood medicine & 7 & 6 & 42 \\
\hline \multirow[t]{3}{*}{ Amaranthaceae } & Alternanthera sessalis $L$. & Dicot & Sessile joy weed & 3 & 2 & 6 \\
\hline & Amaranthus hybridus $L$ & Dicot & - & 11 & 10 & 110 \\
\hline & Amaranthuss spinosus $L$ & Dicot & Spiny amaranth & 13 & 12 & 156 \\
\hline Apiaceae & Centella asiatica (L.) Urb. & Dicot & Penny wort & 5 & 4 & 20 \\
\hline Araceae & Colocasia esculentus $L$ & Dicot & Cocoyam & 8 & 7 & 56 \\
\hline Arecaceae & Raffia fanivera Gaertnb(L) & Monocot & Raffia palm & 19 & 18 & 342 \\
\hline \multirow[t]{5}{*}{ Asteraceae } & Ageratum cornyzoides. $L$ & Dicot & King grass & 22 & 21 & 462 \\
\hline & Tithonia diversifolia Hemsl. & Dicot & Sun flower & 21 & 20 & 420 \\
\hline & Vernonia amygdalina Del & Dicot & Bitter leaves & 16 & 15 & 240 \\
\hline & Vernonia hymenolepis A. Rich & Dicot & Sweet bitter leaves & 14 & 13 & 182 \\
\hline & Spilanthes filicaulis (Schum. \& Thonn.) C. D. Adams & Dicot & Eye for fowl & 18 & 17 & 306 \\
\hline \multirow[t]{2}{*}{ Commelinaceae } & Commelina beghalensis $L$ & Dicot & - & 119 & 118 & 14042 \\
\hline & Commelina diffusa (Burn) $F$ & Dicot & Tropical sidewort & 16 & 15 & 240 \\
\hline \multirow[t]{2}{*}{ Convolvulaceae } & Ipomoea aquatic Forsk & Dicot & - & 14 & 13 & 182 \\
\hline & Ipomea batatas $L$ & Dicot & Sweet potatoes & 5 & 4 & 20 \\
\hline Cyperaceae & Cyperus distans & Monocot & - & 63 & 62 & 3906 \\
\hline Dryopteridaceae & Dryopteris felix-mas(L) Schoot & Dicot & Fern plant & 9 & 8 & 72 \\
\hline Malvacea & Urena lobata $L$ & Dicot & - & 21 & 20 & 420 \\
\hline Maranthaceae & Marantchloa purpurea (Ridl.) Milne-Redh & Dicot & - & 6 & 5 & 30 \\
\hline Nymphaeceae & Nymphea alba $(H)$ & Dicot & Water lily & 8 & 7 & 56 \\
\hline Onagraceae & Ludwigia abysisnica A. Rich & Dicot & Water primerose & 17 & 16 & 272 \\
\hline \multirow[t]{11}{*}{ Poaceae } & Anzonopus compressus (Sw.) P. Beauv. & Monocot & Flat leaf carpet grass & 5 & 4 & 20 \\
\hline & Coix lachrymal-jobi (L) & Monocot & - & 13 & 12 & 156 \\
\hline & Cyndon dactylon (Linn) & Monocot & Bermuda grass & 103 & 102 & 10506 \\
\hline & Echinochloa crus-pavonis (Kunth) Schult & Monocot & - & 20 & 19 & 380 \\
\hline & Echinochloa paramidelis (Lam.) Hitchc & Monocot & - & 43 & 42 & 1806 \\
\hline & Eragrostis spp. Michx. & Monocot & - & 18 & 17 & 306 \\
\hline & mperatea cylindica & Monocot & Spear grass & 10 & 9 & 90 \\
\hline & Leersia hexandra Sw. & Monocot & - & 68 & 67 & 4556 \\
\hline & Panicum maximum Jacq. Var & Monocot & Guinea grass & 19 & 18 & 342 \\
\hline & Paspalum conjugatum P. J. Bergius & Monocot & Sour grass & 21 & 20 & 420 \\
\hline & Pennisetum purpereum (Schumach) & Monocot & Elephant grass & 21 & 20 & 420 \\
\hline Pteridiaceae & Pteridium equilinum & Dicot & Braken fern & 7 & 6 & 42 \\
\hline Polygonaceae & Polygonum limbatum Meisn. & Dicot & - & 47 & 46 & 2162 \\
\hline Portuleraceae & Portuleca oleraceae $L$ & Dicot & - & 4 & 3 & 12 \\
\hline Rubiaceae & Spermacocelatifolia Aubl & Dicot & - & 11 & 10 & 110 \\
\hline Sellaginellaceae & Sellaginella Kraussiana (Kunze)A.Braun & Dicot & - & 2 & 1 & 2 \\
\hline Total & 40 & & & 889 & & 43754 \\
\hline
\end{tabular}


Simpson $=0.94$

Table 5: Simpson index of diversity from vegetation samples at Mbelewa.

Page 10/18 


\begin{tabular}{|c|c|c|c|c|c|c|}
\hline Family & Species & Class & Common name & $\mathbf{n}$ & $n-1$ & $n(n-1)$ \\
\hline \multirow[t]{5}{*}{ Acanthaceae } & Brilantasia nitens Lindau & Dicot & - & 22 & 21 & 462 \\
\hline & Decliptera laxata C. B. Clarke & Dicot & - & 10 & 9 & 90 \\
\hline & Justicia obliquifolia Standl. & Dicot & Blood medicine & 5 & 4 & 20 \\
\hline & Justicia flava (Forssk.) Vahl & Dicot & Blood medicine & 17 & 16 & 272 \\
\hline & Nesonia canenscens Var. Smithii & Dicot & & 7 & 6 & 42 \\
\hline \multirow[t]{3}{*}{ Amaranthaceae } & Alternanthera sessalis $L$. & Dicot & Sessile joy weed & 19 & 18 & 342 \\
\hline & Amaranthus hybridus $L$ & Dicot & - & 20 & 19 & 380 \\
\hline & Amaranthuss spinosus $L$ & Dicot & Spiny amaranth & 6 & 5 & 30 \\
\hline Apiaceae & Centella asiatica (L.) Urb. & Dicot & Penny wort & 5 & 4 & 20 \\
\hline Apocynacea & Catharathus roseus & Dicot & Periwinkle & 20 & 19 & 380 \\
\hline Araceae & Colocasia esculentus $L$ & Dicot & Cocoyam & 4 & 3 & 12 \\
\hline Arecaceae & Raffia fanivera Gaertnb(L) & Monocot & Raffia palm & 27 & 26 & 702 \\
\hline \multirow[t]{5}{*}{ Asteraceae } & Ageratum cornyzoides. $L$ & Dicot & King grass & 24 & 23 & 552 \\
\hline & Vernonia amygdalina Del & Dicot & Bitter leaf & 4 & 3 & 12 \\
\hline & Vernonia gigantean (Walter) Trel. & Dicot & Wild large-leaf bitter leaf & 2 & 1 & 2 \\
\hline & Spilanthes filicaulis (Schum. \& Thonn.) C. D. Adams & Dicot & Eye for fowl & 5 & 4 & 20 \\
\hline & Synedrella nodiflora Cabi. & Dicot & Node weed & 3 & 2 & 6 \\
\hline Athyraccea & Diplazium zammati (Khhn) C. Chr. & Dicot & Single branch fern in fold & 2 & 1 & 2 \\
\hline \multirow[t]{2}{*}{ Commelinaceae } & Commelina beghalensis $L$ & Dicot & - & 19 & 18 & 342 \\
\hline & Commelina diffusa (Burn) $F$ & Dicot & Tropical sidewort & 10 & 9 & 90 \\
\hline \multirow[t]{2}{*}{ Convolvulaceae } & Ipomoea aquatic Forsk & Dicot & - & 17 & 16 & 272 \\
\hline & Ipomea batatas $L$ & Dicot & Sweet potatoes & 7 & 6 & 42 \\
\hline \multirow[t]{4}{*}{ Cyperaceae } & Cyperus haspen & Monocot & - & 21 & 20 & 420 \\
\hline & Cyperus distans & Monocot & - & 16 & 15 & 240 \\
\hline & Mariscus alternifolius (Sensus hepper) & Monocot & - & 14 & 13 & 182 \\
\hline & Rhynchospora corymbosa (L.) Britton. & Monocot & - & 12 & 11 & 132 \\
\hline Dryopteridaceae & Dryopteris felix-mas(L) Schoot & Dicot & Fern plant & 22 & 21 & 462 \\
\hline \multirow[t]{2}{*}{ Fabaceae } & Centrosoma Pubescens (Ridl.) Milne-Redh & Dicot & - & 19 & 18 & 342 \\
\hline & Desmodium salicifolium (Poir.) DC & Dicot & - & 5 & 4 & 20 \\
\hline Malvacea & Urena lobata $L$ & Dicot & - & 20 & 19 & 380 \\
\hline Maranthaceae & Marantchloa purpurea (Ridl.) Milne-Redh & Dicot & - & 6 & 5 & 30 \\
\hline Onagraceae & Ludwigia abysisnica A. Rich & Dicot & Water primerose & 47 & 46 & 2162 \\
\hline Piperaceae & Piper umberlatum (L) & Dicot & - & 4 & 3 & 12 \\
\hline \multirow[t]{8}{*}{ Poaceae } & Coix lachrymal-jobi (L) & Monocot & - & 70 & 69 & 4830 \\
\hline & Cyndon dactylon (Linn) & Monocot & Bermuda grass & 9 & 8 & 72 \\
\hline & Digitaria sanguinalis $(L)$ & Monocot & Crab grass & 16 & 15 & 240 \\
\hline & Echinochloa paramidelis (Lam.) Hitchc & Monocot & - & 97 & 96 & 9312 \\
\hline & Leersia hexandra Sw. & Monocot & - & 63 & 62 & 3906 \\
\hline & Paspalum conjugatum P. J. Bergius & Monocot & Sour grass & 12 & 11 & 132 \\
\hline & Pennisetum purpereum (Schumach) & Monocot & Elephant grass & 3 & 2 & 6 \\
\hline & Saciolepis Africana & Monocot & - & 20 & 19 & 380 \\
\hline \multirow[t]{2}{*}{ Polygonaceae } & Rumex nepalensis $L$ & Dicot & - & 8 & 7 & 56 \\
\hline & Polygonium Limbatum Meisn. & Dicot & - & 12 & 11 & 132 \\
\hline
\end{tabular}




\begin{tabular}{|c|c|c|c|c|c|c|}
\hline Family & Species & Class & Common name & $\mathbf{n}$ & $\mathrm{n}-1$ & $n(n-1)$ \\
\hline Pteridiaceae & Pteridium equilinum & Dicot & Braken fern & 3 & 2 & 6 \\
\hline Rubiaceae & Spermacoce latifolia Aubl. & Dicot & - & 15 & 14 & 210 \\
\hline Sellaginellaceae & Sellaginella Kraussiana (Kunze)A.Braun & Dicot & - & 16 & 15 & 240 \\
\hline Smilalacaceae & Smilax Krausiana Meisn & Dicot & - & 18 & 17 & 306 \\
\hline Solanaceae & Physalis angulate L. var. & Dicot & Fejoo & 2 & 1 & 2 \\
\hline Tilaceae & Triumfetta cordifolia A. Rich. & Dicot & - & 18 & 17 & 306 \\
\hline Zingiberaceae & Aframomum officinalis Schum & Monocot & - & 10 & 9 & 90 \\
\hline Total & 50 & & & 833 & & 28700 \\
\hline
\end{tabular}

$D=0.041411$

Simpson Index $\mathbf{0} 0.96$

\section{Variability of Surface Soil Properties in the Wetlands of Bamenda Municipality and the relationship with macrophyte diversity}

Table 6 shows the mean physico-chemical properties of surface soils in the three major sites assessed. The soils were slightly acidic with a lower pH (1:2.5) water value of 5.35 recorded at the Mulang site (that receives drains from the urban zone) than that of $6.04 \mathrm{pH}$ value recorded at the Mbelewa site (the control site). The soils of the Mulang site had the lowest $\mathrm{pH}$ and the least diversity of plants. The soils of the urban wetland site (Mulang) site registered the highest EC of $309 \mathrm{uS} / \mathrm{cm}$ while the lowest EC value $(120 \mathrm{uS} / \mathrm{cm})$ was obtained at the Peri-urban site of Mile four. Soil organic carbon was in the order of 14.38 $\%>13.88 \%>13.30 \%$ for the Mulang, Mbelewa and Mile Four site, respectively. Organic carbon however showed moderate variation at the control and priurban site but was highly variable at the urban site with a lower diversity of species. Just like the SOM the soils of the urban zone had the best $\mathrm{C} / \mathrm{N}$ ratio of 14.9. The sum of the exchangeable bases across the study sites were similar (0.97, 1.09, 1.00 for the Mbelewa site, Mile Four site and Mulang site, respectively). Exchangeable acidity stood in the order of $0.378>0.300>0.223$ for the control site, peri urban and urban site respectively. The concentration of exchangeable $\mathrm{Al}^{3+}$ was highest at the urban site.

These soils were evaluated for their variability using the coefficient of variation (CV \%). CV values ranging from 0-15\% are considered slightly variable, $15-35 \%$ moderately variable, while $>35 \%$ they are considered highly variable (Tabi et al., 2012; Asongwe et al., 2016). From the data, (Table 7) $\mathrm{Sand}$, $\mathrm{pH}-\mathrm{H}_{2} \mathrm{O}$, $\mathrm{pH}-\mathrm{KCl}$, $\mathrm{Na}$ were consistently least variable across the three sites. $\mathrm{Mg}^{2+}, \Sigma \mathrm{Bases}$, were moderately variable across the three zones. $\mathrm{C} / \mathrm{N}$, and Exch. Acidity, were highly variable.

Table 6: Physico-chemical properties of surface soil properties at the Mile Four, Foncha and Mulang sites of the Bamenda Municipality.

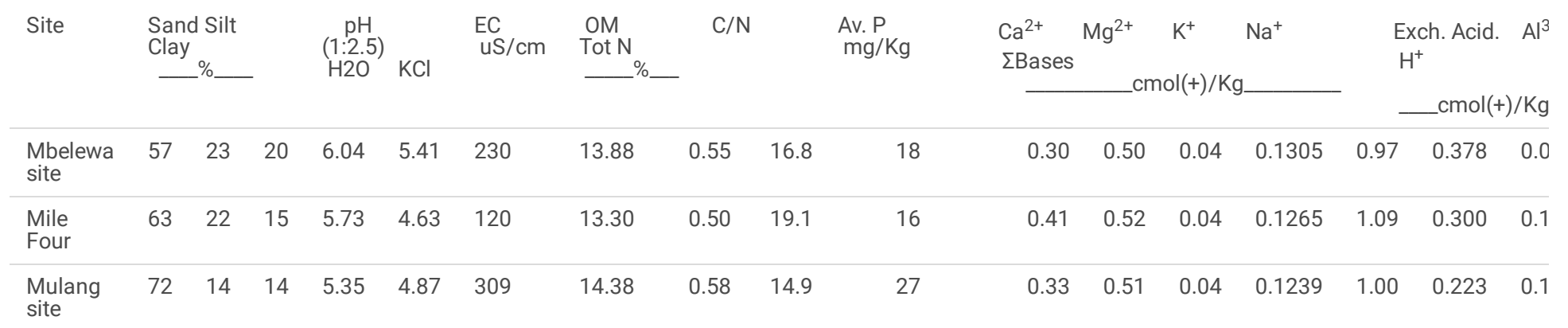

Table 7: Variability classes of soil properties for the different sites in Bamenda municipality.

\begin{tabular}{|c|c|c|c|}
\hline Site & Least variable $(\mathrm{CV}<15 \%)$ & Moderatly Vaiable $(15<\mathrm{CV} \leq 35 \%$ & Highly Variable CV> 35\%) \\
\hline $\begin{array}{l}\text { Mbelewa } \\
\text { site }\end{array}$ & $\begin{array}{l}\text { Sand, silt, clay } \mathrm{pHH}_{2} \mathrm{O}, \mathrm{pHKCl}, \mathrm{EC}, \mathrm{Na}^{+}, \mathrm{Al}^{3+} \text {, } \\
\text { CECsoil, ECEC }\end{array}$ & OM, Tot. $\mathrm{N} \mathrm{Ca}^{2+}, \mathrm{Mg}^{2+}, \mathrm{K}^{+}, \Sigma$ Bases, BSECEC. & C/N, Av. P, Exch.acidity, $\mathrm{H}^{+}$, CECclay. \\
\hline Mile Four & Sand, $\mathrm{pHH}_{2} \mathrm{O}, \mathrm{pHKCl}, \mathrm{K}^{+}, \mathrm{Na}^{+}$, CECsoil, ECEC. & Silt, clay, EC, OM, Av.P, $\mathrm{Mg}^{2+}, \Sigma$ Bases, BSECEC. & Tot. N, C/N, $\mathrm{Ca}^{2+}$, Exch. Acidity, ECECclay \\
\hline $\begin{array}{l}\text { Mulang } \\
\text { site }\end{array}$ & Sand, $\mathrm{pHH}_{2} \mathrm{O}, \mathrm{pHKCl}, \mathrm{Na}^{+}$ & $\begin{array}{l}\text { Silt, Clay, Tot. } \mathrm{N}, \mathrm{Ca}^{2+}, \mathrm{Mg}^{2+}, \mathrm{K}^{+}, \mathrm{Na}^{+}, \Sigma \text { Bases, } \\
\mathrm{Al}^{3+}, \mathrm{CEC} \text { sol, } \mathrm{B}\end{array}$ & $\begin{array}{l}\text { EC, OM, C/N, Av.P, Exch. Acidity, } \mathrm{H}^{+} \text {, } \\
\text { CECclay, BSCEC }\end{array}$ \\
\hline
\end{tabular}

\section{Clustering of the variables}

Table 8 and figure 4 respectively shows the agglomeration table and the hierarchical dendrograms for classification of the chemical variables for the surface soils from the wetlands. Two significant clusters were formed. Amongst the two major clusters, one cluster combined rural environment (control site, site 21) and the peri-urban site where urbanization inputs are minimal. The other cluster represented the urban environment with varying human activities. In the urban 
zone. Alternanthera sessilis was widespread in this cluster. The Mann-Whitney $\mathrm{U}$ test, revealed a significant lower $(\mathrm{P}<0.05)$ concentration of the chemical constituents of the control site and the urban sites indicating contamination, warranting monitoring.

Table 8: Agglomeratin schedule for different clusters obtained from soil analyses in the fresh water wetlands of Bamenda Municipality

\begin{tabular}{|c|c|c|c|c|c|c|}
\hline \multicolumn{7}{|c|}{ Agglomeration Schedule } \\
\hline \multirow[t]{2}{*}{ Stage } & \multicolumn{2}{|c|}{ Cluster Combined } & \multirow[t]{2}{*}{ Coefficients } & \multicolumn{2}{|c|}{ Stage Cluster First Appears } & \multirow[t]{2}{*}{ Next Stage } \\
\hline & Cluster 1 & Cluster 2 & & Cluster 1 & Cluster 2 & \\
\hline 1 & 14 & 18 & .002 & 0 & 0 & 4 \\
\hline 2 & 23 & 24 & .010 & 0 & 0 & 12 \\
\hline 3 & 17 & 19 & .015 & 0 & 0 & 5 \\
\hline 4 & 14 & 15 & .081 & 1 & 0 & 7 \\
\hline 5 & 12 & 17 & .338 & 0 & 3 & 7 \\
\hline 6 & 9 & 13 & .561 & 0 & 0 & 9 \\
\hline 7 & 12 & 14 & .840 & 5 & 4 & 9 \\
\hline 8 & 20 & 22 & 1.192 & 0 & 0 & 19 \\
\hline 9 & 9 & 12 & 1.681 & 6 & 7 & 11 \\
\hline 10 & 4 & 5 & 1.820 & 0 & 0 & 12 \\
\hline 11 & 9 & 16 & 7.061 & 9 & 0 & 14 \\
\hline 12 & 4 & 23 & 30.756 & 10 & 2 & 14 \\
\hline 13 & 2 & 3 & 112.000 & 0 & 0 & 17 \\
\hline 14 & 4 & 9 & 129.863 & 12 & 11 & 18 \\
\hline 15 & 7 & 8 & 399.432 & 0 & 0 & 16 \\
\hline 16 & 7 & 10 & 849.348 & 15 & 0 & 17 \\
\hline 17 & 2 & 7 & 908.425 & 13 & 16 & 18 \\
\hline 18 & 2 & 4 & 2340.274 & 17 & 14 & 20 \\
\hline 19 & 11 & 20 & 5437.587 & 0 & 8 & 20 \\
\hline 20 & 2 & 11 & 9244.048 & 18 & 19 & 22 \\
\hline 21 & 1 & 21 & 26015.207 & 0 & 0 & 22 \\
\hline 22 & 1 & 2 & 41940.680 & 21 & 20 & 23 \\
\hline 23 & 1 & 6 & 732950.182 & 22 & 0 & 0 \\
\hline
\end{tabular}

\section{Discussions}

From the floristic study, semi aquatic and marshland plant species in the wetlands were mainly herbaceous and shrubby and showed discoloration in patches throughout the wetland. According to Tsala et al., (1993) and Halabowski et al., (2020), the presence of metal pollutants in wastewater and other anthropogenic influences constitute a stress factor for plants through their toxic effects on the photosynthetic process and membrane function. The decolouration of vegetation in this wetland could therefore be allied to excessive chemical elements from anthropogenic activities.

The study sites did not differ considerably from the rest as they clustered together as a community, probably due to high similarity in species composition that are affected by similar levels of environmental disturbances. Human activities involving cutting, of raffias and woody species resulted to an open vegetation with shrubs scattered all over the wetlands. Hyde and Wursten (2007) and Zelnik et al., (2021) have noted similar vegetation composition in the miningimpacted sites in wetlands along Lake Victoria in East Africa and Slovenian watercourses, respectively. This could be indicative that, the areas have suffered some degree of disturbance either from cultivation or other human activities with the exception of the control site, with a relatively higher number of trees.

Macrophytes constituted an important part of the wetland. According to Lindholm et al., (2020), macrophytes play a key role in the remediation of macro and micronutrients because they have the capacity to grow vigorously in nutrient rich environments especially emergent and floating species. The plants could absorb and sequester pollutants; or reduce erosion by damping wave actions and as such should be considered for proper management in order to contribute significantly to the wellbeing of other components of the dynamic aquatic system.

Quadrat studies revealed 50 plant (macrophytes) species (mainly emergent herbaceous plants: grasses) distributed in 28 families. Ahmad et al., (2009) reported that grasses which are represented by 10.000 species and about 610 genera are the most widespread family of flowering plants of the world. Just as 
any aquatic ecosystem, these grasses were dominated by monocots. In some ponded areas close to the main river course, floating macrophytes were observed. Tita et al., (2012) did not identify such plants in the urban segment of the Mezam River system nor made mention of them in ponded areas proxy to it. The latter reported that in 2007, the Nkoup River system was characterized by eutrophic species such as Potamogeton spp and Ceratophyllum demersum in the upstream segments considerably impacted by agriculture whereas the downstream and urban segments were dominated by floating and emergent species all of which accumulate and bio-concentrate significant amounts of metal pollutants. The fact that these plants were not reported in the agricultural wetlands in the Bamenda Municipality is an indication that this wetland is under stress activities and, thus continually degrading. Diverse urban amenities with some having considerably noxious activities have come to existence within the environs of this agricultural wetland of the municipality. These activities either impinge directly through physical alteration and development and/or indirectly through widespread diverse chemical inputs on the agricultural soils. Acho-Chi (1998) had commented on this alteration.

In the wetlands of Bamenda Municipality, Poaceae (26\%) occurred as the most represented family, followed by Asteraceae (12\%), Cyperaceae (8\%), Acanthaceae (6\%), Amaranthaceae (6\%) with other species less represented. The marsh land from visual observation was dominated by Leersia hexandra growing in open water and covering large areas. In the area Pennicitum purpureum and or Tithonia diversifolia lined the main river course with doted spots of Sacharum sp., Coix sp, Ludwigia, sp, and Nesonia sp.

From the flora distribution, the control site at Mbelewa showed the greatest level of species diversity. Species biodiversity is used to indicate the 'biological health' of a particular habitat and is higher in less polluted areas. The control site at Mbelewa was represented by 39 species. Here, the dominant species ranked in abundance were as follows Raphia farinifera > Ludwigia hexandra > Coix spp.> Leersia hexandra > Ehchinochloa paramidelis.

The peri-urban site (Mile 4 to Below Foncha segment) which was represented by 40 species had the dominant species ranked in abundance as Commelina bengalensis > Leersia hexandra > Cyperus distance > Ehchinochloa pyramidalis. The dominance of Commelina benghalensis generally occurring in extensive uniform stands along the edge of the channel and in ponded and pond- like areas was similarly reported by Tita et el., (2012). These plants thrive well in seasonally flooded environments and as such their abundance and diversity is proof of their high tolerance for fluctuating water levels and anthropogenic disturbances in the area (Grosshans and Kenkel, 1997, Lindholm et al., 2020).

At the Ayaba to Ngomegham segment (at Mulang), Pennisetum purpureum $>$ Echinochloa pyramidalis $>$ Tithonia diversifolia $>$ Leersia hexandra were the abundant species. Pennisetum purpureum, Echinochloa pyramidalis and Tithonia diversifolia lined the main river course. Amongst the plants found in the area, Coix sp, Poligonium sp, Ludwigia sp, Nesonia sp, Leersia sp, Echinochloa sp, Portulaca oleraceae are obligate wetland species and thus rarely occur on dry land, while Penicum sp and Pennisetum purpureum have the same likelihood of occurring in wetlands as on dry land. Portulaca oleracea is a weed in cultivated fields and waste moist marshy places. It is also used as a vegetable and medicinal herb (Sher et al., 2011; Marwat et al., 2011). Penniseteum purpureum amongst others is very effective in waste water treatment (Greenway, 2003; and Tita et al., 2012). Alternanthera sessilis is a common species, very widespread in waste and cultivated grounds, especially in damp or wet conditions (Townsend, 1974). It is an agricultural weed that invades disturbed wet areas in tropical and subtropical areas. Overrall, $A$. sessilis has a low significant ecological impact (Tomaino, 2006) and thus a good indicator species. Just as, A. sessilis, Rumex dentatus L., apart from its allelopathic activity (producing substances that inhibit the growth of other plants near it), it also grows in disturbed habitats, often in moist areas.

Lower Simpson indices of diversity (0.94) recorded at the urban and peri-urban indicates that species diversity at the control site is higher than that at the urban and peri-urban sites that show no differences. The urban site had lower species abundances in certain areas, indicating worse environmental conditions such as from toxic chemical inputs from the urban catchment. Similar findings due to worse environmental conditions have been reported by Germ et al., (2021) in a study to assess the diversity of macrophytes and environment of the Ljubljanica River (Slovenia).

\section{Variability of Surface Soil Properties in the Wetlands of Bamenda Municipality and the relationship with macrophyte diversity}

The soils were slightly acidic with a lower $\mathrm{pH}(1: 2.5)$ water value of 5.35 recorded at the urban site at Mulang (that receives drains from the urban zone) than that of $6.04 \mathrm{pH}$ value recorded at the Mbelewa site (the control site). Soil pH plays a primordial role in nutrient availability to plants and therefore their growth, development and diversity. Low pH reduces the mineralization of soil organic matter and other nutrient reserves, inhibiting root growth and consequently, adsorption of nutrients. The soils of the Mulang site had the lowest $\mathrm{pH}$ and the least diversity of plants. The acidic nature of the soil at this site can also be attributed to various anthropogenic factors. The soils of the urban wetland site (Mulang) site registered the highest EC of 309 uS/cm while the lowest EC value $(120 \mathrm{uS} / \mathrm{cm})$ was obtained at the Peri-urban site of Mile four. The highest pH recorded in the urban site could be associated to additional inputs ions from industrial urban activities. Soil organic carbon was in the order of $14.38 \%>13.88 \%>13.30 \%$ for the Mulang, Mbelewa and Mile Four site, respectively. Soil organic matter is a good indicator of soil fertility and one of the most important soil components, along with stabilization soil structure and improving infiltration rate. Nowadays, soil organic matter stabilization is perceived as a mechanism for organic carbon storage in the soil. Organic matter supplies energy and cell building constituents for most microorganisms and is a critical factor in soil fertility. Organic carbon however showed moderate variation at the control and pri-urban site but was highly variable at the urban site with a lower diversity of species. Just like the SOM the soils of the urban zone had the best $\mathrm{C} / \mathrm{N}$ ratio of 14.9 . This means that the mineralisation rate of the SOM is good. The sum of the exchangeable bases across the study sites were similar $(0.97,1.09,1.00$ for the Mbelewa site, Mile Four site and Mulang site, respectively). Exchangeable acidity stood in the order of $0.378>0.300>0.223$ for the control site, peri urban and urban site respectively. However, the concentration of exchangeable $\mathrm{Al}^{3+}$ was highest at the urban site, and a probable indication of inputs from industrial inputs from the urban site.

From the results, Sand, $\mathrm{pH}-\mathrm{H}_{2} \mathrm{O}, \mathrm{pH}-\mathrm{KCl}, \mathrm{Na}$ were consistently least variable across the three sites. Tabi and Ogunkunle (2007) had similarly reported least variability of soil $\mathrm{pH}$ for vertisols under rice cultivation in the Logone flood plain of Northern Cameroon. A very important parameter that influences many physico-chemical properties of soils including the availability of nutrients, plants richness and diversity is soil pH. In this study, though the pH variability reported is small, minor changes in $\mathrm{pH}$ units have significant effects on nutrient availability. Moderately variable $\mathrm{Mg}^{2+}$ and $\Sigma$ Bases were observed across the 
three zones of the study area which could be attributed to variation in levels of alluvial materials received. Also, variation in chrono sequences of materials that have been subjected to different intensities of weathering could have a significant effect on these physical parameters. All these factors have significant implications on nutrient availability to plants of the wetlands. The moderate variability of these bases implies that, for proper management of the wetlands, a unique policy for the area is insufficient to conserve the wetlands. $\mathrm{C} / \mathrm{N}$, and Exch. Acidity, were highly variable. These parameters owe a lot of their origin to organic materials, urban swept off, and farm inputs that could have also influenced the vegetation diversity across the zones.

\section{Clustering of the variables}

From the hierarchical dendrograms for the classification of the chemical variables of the surface soils from the wetlands, two significant clusters were formed. The clusters correspond to the geographical location of the sampling sites and possible sources defining the soil quality like agricultural activity, industrial impact and fertilizing, which influences the diversity of macroflora in the area. Amongst the two major clusters, one cluster combined rural environment (control site) and the peri-urban site where urbanization inputs are minimal. The other cluster represented the urban environment with varying human activities. In the urban zone. Alternanthera sessilis which was widespread in this cluster is a common species, very extensive in waste and cultivated grounds, especially in damp or wet conditions (Townsend, 1974). According to Lazzaro et al. (2020), it impacts native plant species. The cluster on the lower part of the dendrogram represents dominantly rural and peri-urban areas with natural origins. Here, the dominant species were ranked in abundance as Commelina bengalensis > Leersia hexandra > Cyperus distance > Ehchinochloa pyramidalis. The dominance of Commelina benghalensis generally occurred in extensive uniform stands. The Mann-Whitney $U$ test, revealed a significant lower $(P<0.05)$ concentration of the chemical constituents of the control site and the urban sites indicating contamination, warranting monitoring.

\section{Conclusions}

The wetlands of the Bamenda municipality have a total documented number of 50 macrophytes species distributed in 28 families. The species observed were mainly emergent herbaceous plants (grasses) with only few shrubs and trees. The Simpson indices of diversity stood at 0.94 and 0.96 for the urban and control sites, respectively. The soils were slightly acidic. Sand, $\mathrm{pH}-\mathrm{H}_{2} \mathrm{O}, \mathrm{pH}-\mathrm{KCl}$, Na were consistently least variable across the three sites. Two significant clusters representing a combination of urban and peri urban/rural were formed from the hierarchical dendrograms for classification of the chemical variables for the surface soils wetlands with associations to plants species. The Mann-Whitney U test, revealed a significant lower $(P<0.05)$ concentration of the chemical constituents of the control site and the urban sites indicating contamination, warranting monitoring.

\section{Declarations}

\section{Acknowledgements}

The authors acknowledge financial support from the Cameroon Ministry of Higher Education through the Research Modernisation Allowance.

\section{Authors' contribution}

This work was carried out in collaboration between all authors. Author AGA, IBB, and AST, designed the study and the data collection instruments, collected the data, performed the statistical analyses and co-wrote the draft with the assistance of Author AEO, VAT, and KPB All read and approved the final manuscript.

\section{Competing Interests}

Authors have declared that no competing interests exist.

\section{References}

1. Ahmad, F.M., Khan, A., Ahmad, M., Zafar, M., Nazir, A., and Marwat, S.K. Taxonomic studies of grasses and their indigenous uses in the salt range area of Pakistan. African Journal of Biotechnology 8 (2), 231-249 (2009).

2. Arruda, D.M., Schaefer, C.E.G.R., Correa, G.R. Landforms and soil attributes determines the vegetation structure in the Brazilian semiarid. Folia Geobot 50, 175-84 (2015)..

3. Baldeck, C.A., Harms, K.E., Yavitt, J.B. Soil resources and topography shape local tree community structure in tropical forests. Procedure for Social, Behavioral and Biological Science (11), 280:301(2013).

4. Borgwardt, F., Robinsonm, L., Trauner, D., Teixeira, H., Nogueira, A.J.A., Lillebø, A.I., Piet, G. Kuemmerlen, M., O’Higgins, T., McDonaldg, H. Exploring variability in environmental impact risk from human activities across aquatic ecosystems. Science of the Total Environment 652, 1396-1408 (2019).

5. Dale, M.R.T. Spatial Pattern Analysis in Plant Ecology. Cambridge,UK: Cambridge University Press. (1999).

6. Germ, M., Janež, V., Gaberš cik, A., Zelnik, I. Diversity of Macrophytes and Environmental Assessment of the Ljubljanica River (Slovenia). Diversity 13, 278291 (2021). https://doi.org/10.3390/d13060278

7. GP-DERUDEP. Grassfield Participatory: Decentralized and Rural Development Project. Baseline study of the North West Province. SIRDEP Bamenda Cameroon, 298pp (2006).

8. Greenway, M. Suitability of macrophytes for nutrient removal from surface flow constructed wetlands receiving secondary treatment effluent in Queensland, Australia. Water Science and Technology 48, 121-128 (2003).

9. Grosshans, R.E. and Kenkel, N.C. Dynamics of emergent vegetation along natural gradients of water depth and salinity in a prairie marsh: delayed influences of competition. UFS Delta Marsh Annual Report 32, 83- 93 (1997). 
10. Guerra, T.N.F., Rodal, M.J.N., Silva, A.C.B.L. Influence of edge and topography on the vegetation in an Atlantic Forest remnant in northeastern Brazil. Journal of Forest Resources 18, 200-208 (2013).

11. Halabowski, D., Lewin, I. Impact of anthropogenic transformations on the vegetation of selected abiotic types of rivers in two ecoregions (Southern Poland). Knowledge Management and Aquatic Ecosystem 10, 200-208 (2020).

12. Hyde, M. and Wursten, B. Flora of Zimbabwe: (2007). htpp://www.Zimbabweflora.co.zw Speciesdata/about.php

13. Lawniczak, A.E., Zhierska, J., Choinski, A., Szczepaniak, W. Response of emergent marcophytes to hydrological changes in a shallow lake with special reference to nutrient cycling. Hydrobiologia 656, 243- 254 (2010).

14. Lazzaro, L., Bolpagni, R., Buffa, G., Gentili, R., Lonati, M., Stinca, A., Acosta, A.T.R., Adorni, M., Aleffi, M., Allegrezza, M. Impact of invasive alien plants on native plant communities and Natura 2000 habitats: State of the art, gap analysis and perspectives in Italy. Journal of Environmental Management 274, 111-140 (2020).

15. Lindholm, M., Alahuhta, J., Heino, J., Hjort, J., Toivonen, H. Changes in the functional features of macrophyte communities and driving factors across a 70-year period. Hydrobiologia, 847, 3811-3827 (2020).

16. Malida, U.N. Water pollution and disposal of waste water on land. Mcgraw-Hill Public Company. New Deilhi, India 628p (1981).

17. MINEP. Ministry of Environment and Nature ProtectionLaw No 96/12 of 05th August 1996 Relating to Environmental Management in Cameroon. Ministry of Environment and Nature Protection of Cameroon, 329 pp. (1996).

18. Ndenecho EN. (2005). Biological Resource Exploitation in Cameroon: From crises to sustainable management. Unique Printers Bamenda Cameroon: $181 \mathrm{pp}$.

19. Pena-Claros, M., Poorter, L., Alarcon. A. Soil effects on forest structure and diversity in a moist and a dry tropical forest. Biotropic 44, 276-83 (2012).

20. Riss, T., and Sand-Jensen, P. Development of vegetation and environmental conditions in an oligotrophic Danish lake over 40 years. Freshwater Biology 40, 123-134 (1998).

21. Solbrig, O.T. The theory and practice of the science of biodiversity: a personal assessment. In: Kato M (ed.). The biology of biodiversity. Springer, Berlin. 9, 107- 117. (2000).

22. Struyf, E, Bal, K., Backx, H., Vrebos, D., Casteleyn, A., De Deckere, E., Schoelynck, J., Berendonck, L., Raitt, L., Meire, P. Nitrogen, phosphorus and silicon in riparian ecosystems along the Berg River (South-Africa): the effect of increasing human land use. Water SA, 38(4), $597-606$ (2012).

23. Tabi, F.O., and Ogunkunle, A.O. Spatial variation of some soil physic-chemical properties of an Alfisol in Southwestern Nigeria. Nigeria Journal of Soil and Environmental Resources 7, 82-91. (2007).

24. Tabi, F.O., Omoko, M., Boukong, A., Mvondo Ze, A.D., Bitondo, D, and Fuh-Che, C. Evaluation of Lowland rice (Oryza sativa) production system and management recommendations for Logone and Chari flood plain-Republic of Cameroon. Agricultural Science Research Journals 2(5), 261-273. (2012).

25. Tita MA, Magha A, and Kamgang KVB (2012). Occurrence of macrophytes in the Mezam river system in Bamenda (Cameroon) and their role in nutrient retention. Syllabus Review, Science Service 3: 1 - 10

26. Todd, D. K. Groundwater Hydrology. John Willey and Sons, New York, USA. (1980).

27. Tomaino A Alternatera sessalis. Invasive species assessment protocol: U.S. national Assessment (2006). Available from

28. http://www.fs.fed.us/r9/wildlife/nnis/documents/invasive_species_assessment_forest<service_region9.pdf.

29. Townsend, C.C. Amaranthaceae. In: Flora of West Pakistan, (Eds.): E. Nasir and S.I. Ali Islamabad 71, 38 - 41 (1974).

30. Tsala, N.G., Omokoo, N.D., and Tita, M.A. Effect of some heavy metals on photosynthetic activity and chlorophyll content in Pistia stratiotes. Journal of Zhejiang Agriculture University 19 (1), 110-116 (1993).

31. Yerima, B.P.K. and Van Ranst, E. Introduction to Soil Science, soil of the Tropics. TRAFFORD Publishers, Victoria Canada, 397pp (2005).

32. Zelnik, I., Kuhar, U., Holcar, M., Germ, M., Gaberš cik, A. Distribution of vascular plant communities in Slovenian watercourses. Water 13, 1071 (2021).

\section{Figures}




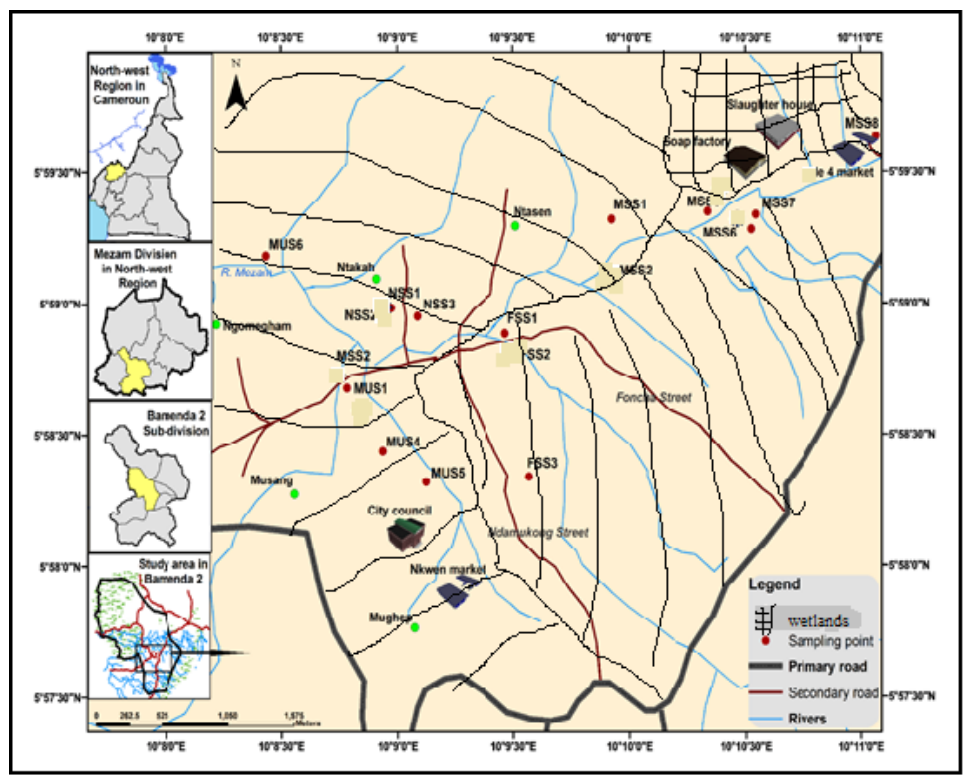

Figure 1

Map of the study area in fresh water wetlands of Bamenda Municipality. Adapted from the 1980 land use map of the Bamenda City Area: Source Bamenda City Council.

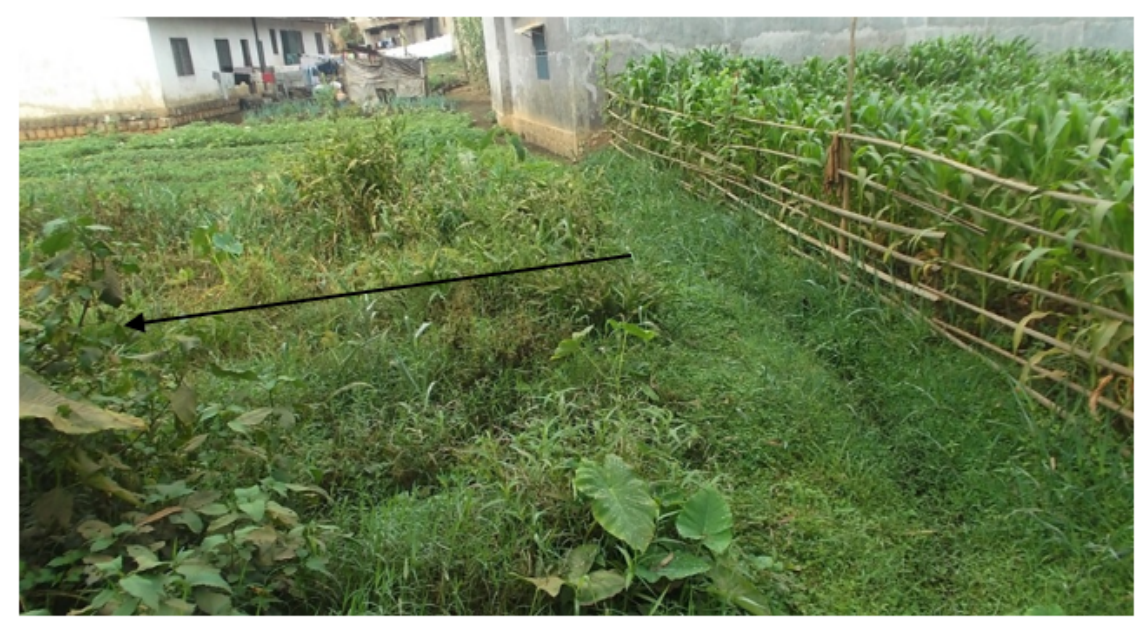

\section{Figure 2}

Discoloration of vegetation along a waste channel in the wetland. 


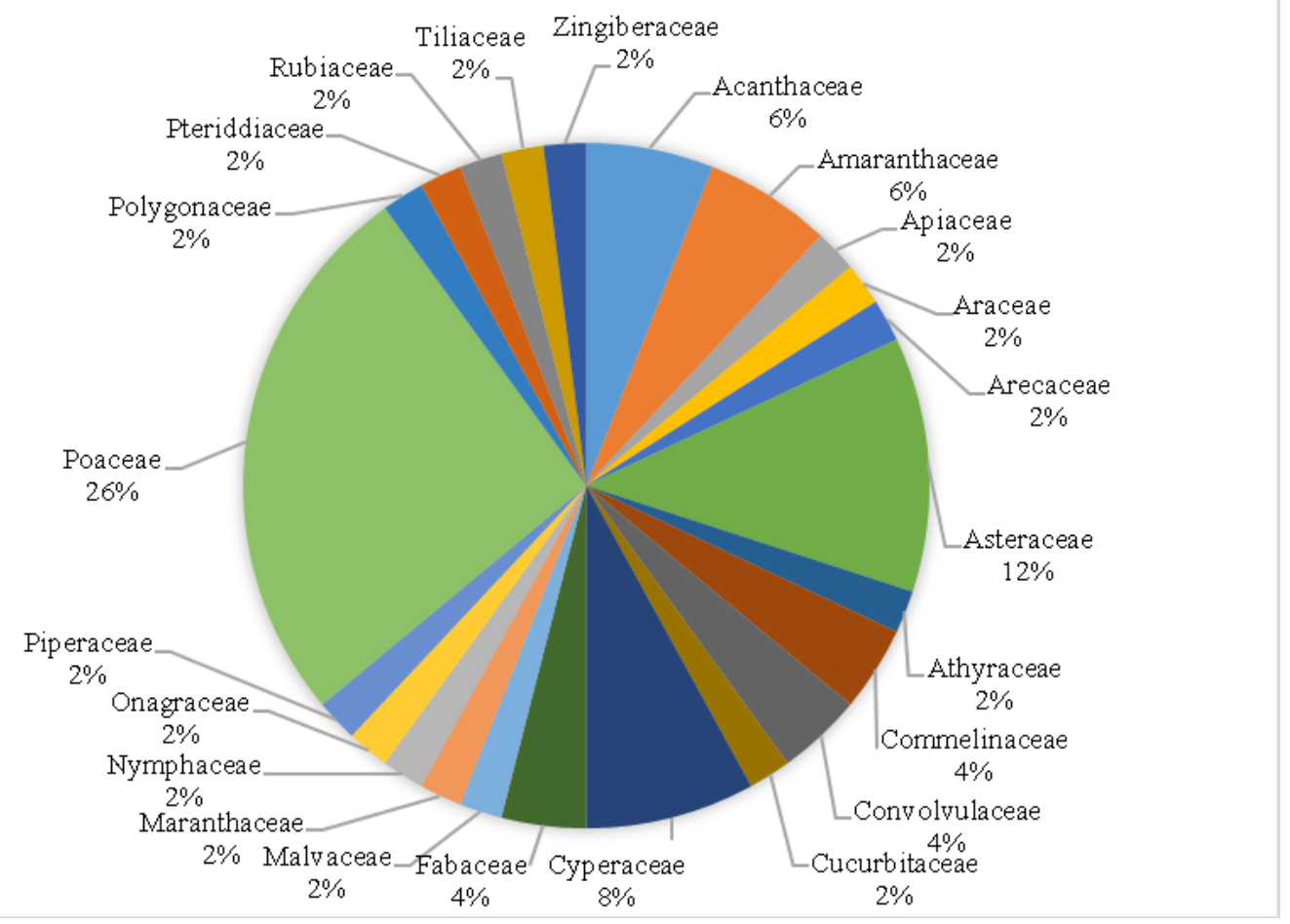

\section{Figure 3}

Percent distribution of riverine wetland flora families in Bamenda Municipality

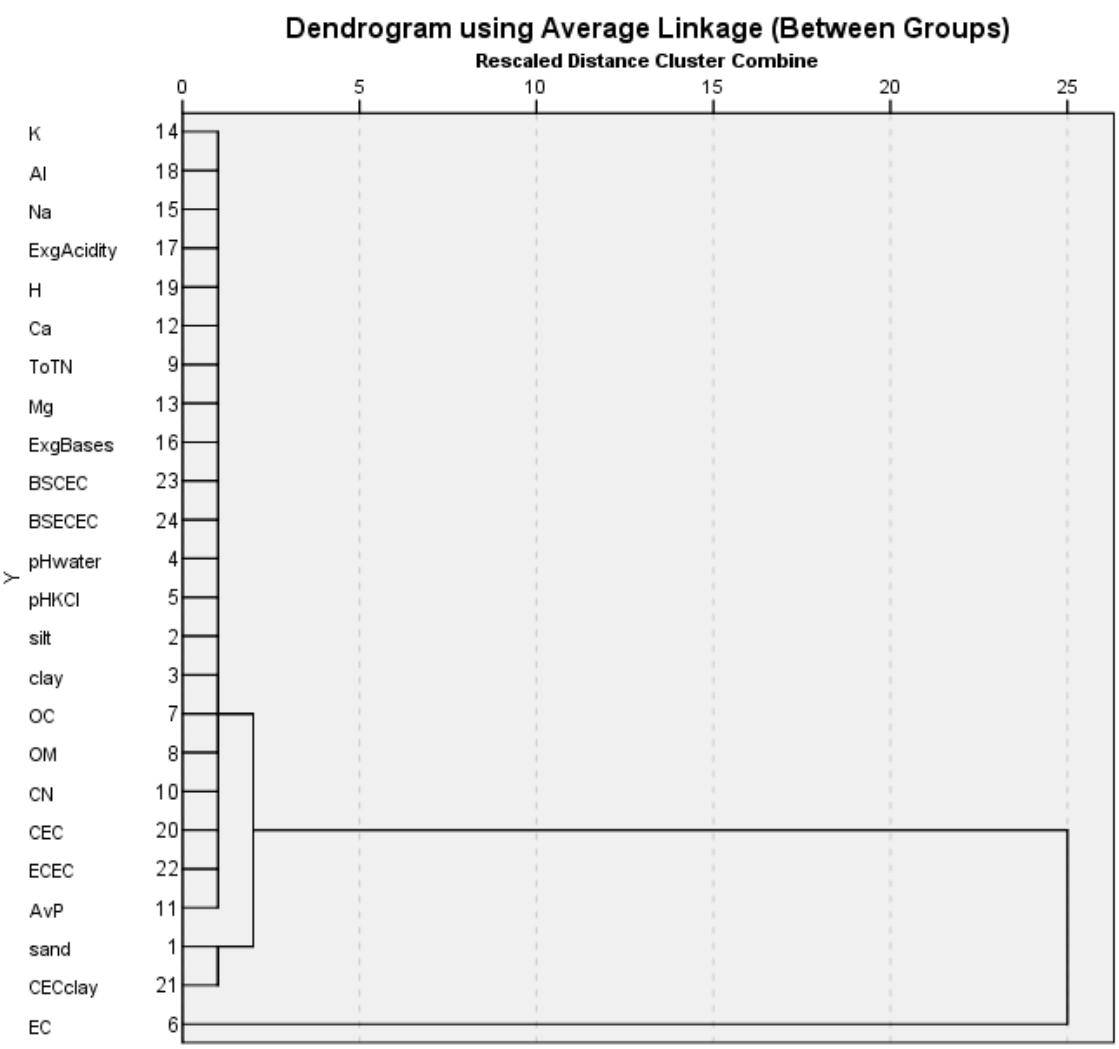

\section{Figure 4}

Dendrogram obtained from soil analyses in the fresh water wetlands of Bamenda Municipality 